\title{
Influences of Strain Rate and Temperature Variation on Material Intrinsic Length of Plasticity Strain Gradient Theory
}

\author{
Ya-Pu Zhao ${ }^{1}$ and T.X. Yu ${ }^{2}$ \\ ${ }^{1}$ LNM, Institute of Mechanics, Chinese Academy of Sciences, 15 Zhong Guan Cun Road, \\ Beijing 100080, P.R. China \\ ${ }^{2}$ Department of Mechanical Engineering, Hong Kong University of Science and Technology, \\ Clear Water Bay, Pokfulam Road, Kowloon, Hong Kong SAR, P.R. China
}

Keywords: Material Intrinsic Length, Scale Effect, Strain Gradient Plasticity, Strain Rate Effect, Temperature Variation

\begin{abstract}
Cowper-Symonds and Johnson-Cook dynamic constitutive relations are used to study the influence of both strain rate effect and temperature variation on the material intrinsic length scale in strain gradient plasticity. The material intrinsic length scale decreases with increasing strain rates, and this length scale increases with temperature.
\end{abstract}

\section{INTRODUCTION}

Recent micro-scale experiments have shown that materials display strong scale effects when the characteristic length scale associated with non-uniform plastic deformation is in the order of microns. Scale effect can not be predicted by the classical plastic constitutive relations, since the existing models possess no internal length scale. The material constant with dimension of length for the constitutive relation of strain gradient effect is called the material intrinsic length. The strain gradient effect is important when the characteristic dimension of the deformation zone is of the same order to the material intrinsic length. Generally speaking, the material intrinsic length is of the order of microns for commonly used materials [1].

The characteristic dimension of micro-electro-mechanical systems (MEMS) components (for example, width of cantilever beams in typical micro-machined accelerometers) is of the order of several microns [2]. Hence, strain gradient effect is important for the mechanical behaviors of MEMS materials [3]. As a kind of inertia device, micro-machined accelerometers may experience very high level of acceleration (say, up to $10^{6} \mathrm{~g}$ ), being often called "high-g accelerometers". High-g accelerometers may also work at the circumstance of large temperature gradient. Therefore, both strain rate effect and temperature variation are crucial to the reliability of such inertia micromachined devices.

The classical dynamic plasticity theory can not predict scale effect, too. No investigation has been carried out for the influence of strain rate effect and temperature variation on the strain gradient plasticity. The objective of the present paper is to study the influence of these two effects on the material intrinsic length. 


\section{MATERIAL INTRINSIC LENGTH}

Nix and Gao [1] derived the following indentation law for strain gradient plasticity

$$
\left(\sigma / \sigma_{0}\right)^{2}=1+\bar{l} \chi
$$

where $\sigma$ is the effective flow stress in the presence of a strain gradient, $\sigma_{0}$ is the flow stress in the absence of a gradient, $\chi$ is the effective strain gradient and $\vec{l}$ is the material intrinsic length, which is, in turn, related to the flow stress of the material in the absence of a strain gradient

$$
\bar{l}=b\left(\mu / \sigma_{0}\right)^{2}
$$

where $b$ is the Burgers vector, $\mu$ is the elastic shear modulus. The quantity $\bar{l}$ is recognized as a length scale with which any strain gradient must be compared to determine the effect of the strain gradient on the flow stress. Usually, the characteristic material length scale is in the order of a few to tens of microns. It is noted that $\bar{l}$ is not constant for a given material but is required to change as the flow stress in the absence of strain gradient changes. In particular, in ordinary strain hardening the flow stress rises with increasing homogeneous strain, thus causing $\bar{l}$ to decrease according to equation (2). For most metals, the yield stress increases with strain rate, while the motion of dislocations is constrained by phonon drag. For the dynamic fracture problems, the plastic zone ahead of a crack will decrease with increasing yield stress. For small scale yielding (SSY), the characteristic dimension of the plastic zone ahead of the crack tip is of the order of microns. Therefore, the consideration of the strain gradient effect in dynamic fracture becomes more necessary.

\section{INFLUENCE OF STRAIN-RATE EFFECT BY COWPER-SYMONDS RELATION}

It is well known that the flow stress of most metallic materials increases with strain rate. Cowper and Symonds [4] suggested the following constitutive relation for the dynamic flow stress

$$
\sigma_{0}^{\prime} / \sigma_{0}=1+(\dot{\varepsilon} / D)^{1 / 4}
$$

where $\sigma_{0}^{\prime}$ denotes the dynamic flow stress at an uniaxial plastic strain rate $\dot{\varepsilon}, D$ and $q$ are constants for a particular material, which have been determined for the materials listed in Table 1 .

Table 1 Coefficients in equation (3)

\begin{tabular}{cccc}
\hline Material & $D\left(s^{-1}\right)$ & $q$ & Reference \\
\hline Mild steel & 40.4 & 5 & Cowper \& Symonds [4] \\
Aluminum alloy & 6500 & 4 & Bodner \& Symonds [5] \\
$\alpha$-Titanium (Ti 50 A) & 120 & 9 & Symonds \& Chon [6] \\
Stainless steel 304 & 100 & 10 & Forrestal \& Sagartz [7] \\
\hline
\end{tabular}


For dynamic plasticity problems, the material intrinsic length can be written as

$$
\bar{l}=b\left(\mu / \sigma_{0}^{\prime}\right)^{2}
$$

Substituting (3) into the above equation yields a dimensionless length scale as follows

$$
\frac{\vec{l}}{b}\left(\frac{\sigma_{0}}{\mu}\right)^{2}=\left[1+\left(\frac{\dot{\varepsilon}}{D}\right)^{1 / q}\right]^{-2}
$$

Thus, the material intrinsic length decreases with increasing strain rate for most metallic materials, as shown in Fig. 1.

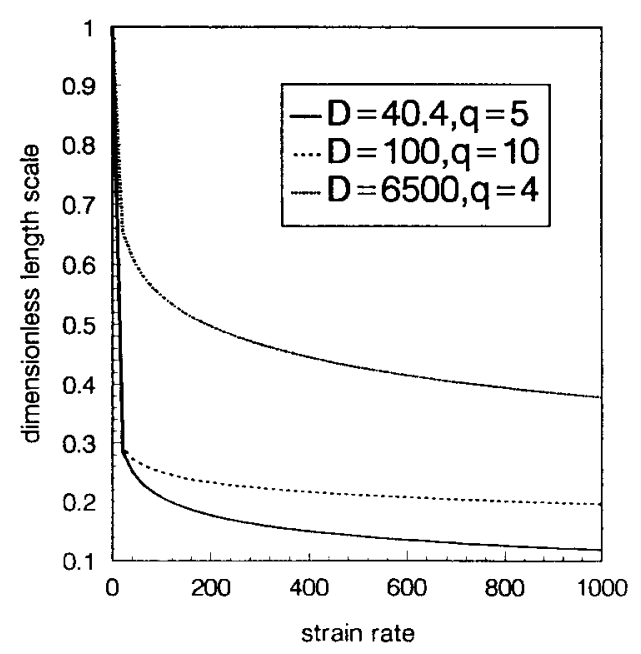

Fig. 1 Variation of dimensionless length scale with strain rate

\section{INFLUENCES OF BOTH STRAIN-RATE EFFECT AND TEMPERATURE VARIATION BY JOHNSON-COOK RELATION}

Many constitutive equations have been developed to describe the behavior of materials under dynamic loading. Some of these models describe only the variation of yield stress with strain rate changes, whilst others describe strain and strain rate hardening effects without softening effect caused by temperature. Johnson and Cook [8,9] proposed the following equation with a term representing temperature softening

$$
\sigma=\left(A+B \varepsilon^{n}\right)\left(1+C \ln \dot{\varepsilon}^{*}\right)\left(1-T^{* m}\right),
$$

where $\varepsilon$ is the equivalent plastic strain, $\dot{\varepsilon}^{*}=\dot{\varepsilon} / \dot{\varepsilon}_{0}$ is the dimensionless plastic strain rate taking $\dot{\varepsilon}_{0}=1 \mathrm{~s}^{-1}$, and $T^{*}=\left(T-T_{r}\right) /\left(T_{m}-T_{r}\right)$ is the homologous temperature, with $T$ being the immediate absolute temperature of the deformed specimen, $T_{r}$ and $T_{m}$ being the room temperature and the melting point, respectively. $A, B, C, n$ and $m$ are yield strength, work hardening coefficient, 
work hardening exponent, strain rate sensitivity and thermal softening coefficient, all being material constants. The expression in the first bracket on the r. h. s. of (5) gives the stress as a function of strain for $\dot{\varepsilon}^{*}=1.0$ and $T^{*}=0$. The expressions in the second and third brackets represent the effects of strain rate and temperature, respectively. The Johnson-Cook equation is a highly useful and successful constitutive model. One of the problems with this equation is that all parameters are coupled by being multiplied to each other. Nevertheless, the Johnson-Cook equation remains the "workhorse" of constitutive modeling [10]. The constants in the Johnson-Cook equation for TiAl have been found to be as follows [11]: $A=290 \times 10^{6}(\mathrm{MPa}), B=1.77 \times 10^{9}(\mathrm{MPa}), \quad n=0.55$, $C=0.006$ and $m=2.787$. The specific constants are listed in Table 2 [9].

Table 2 Summary of strength constants [9]

\begin{tabular}{cccccc}
\hline Material & $A(\mathrm{MPa})$ & $B(\mathrm{MPa})$ & $n$ & $C$ & $M$ \\
\hline OFHC copper & 90 & 292 & 0.31 & 0.025 & 1.09 \\
Cartridge brass & 112 & 505 & 0.42 & 0.009 & 1.68 \\
Nickel 200 & 163 & 648 & 0.33 & 0.006 & 1.44 \\
Armco iron & 175 & 380 & 0.32 & 0.060 & 0.55 \\
Carpenter Electric iron & 290 & 339 & 0.40 & 0.055 & 0.55 \\
1006 steel & 350 & 275 & 0.36 & 0.022 & 1.00 \\
2024-T351 aluminum & 265 & 426 & 0.34 & 0.015 & 1.00 \\
7039 aluminum & 337 & 343 & 0.41 & 0.010 & 1.00 \\
4340 steel & 792 & 510 & 0.26 & 0.014 & 1.03 \\
S-7 tool steel & 1539 & 477 & 0.18 & 0.012 & 1.00 \\
Tungsten alloy & 1506 & 177 & 0.12 & 0.016 & 1.00 \\
(.07Ni, .03Fe) & & & & & \\
Depleted uranium & 1079 & 1120 & 0.25 & 0.007 & 1.00 \\
-0.75 Ti & & & & & \\
\hline
\end{tabular}

If we neglect the strain-hardening effect, then Johnson-Cook equation is reduced to

$$
\sigma=\sigma_{0}\left(1+C \ln \dot{\varepsilon}^{*}\right)\left(1-T^{* m}\right)
$$

where $\sigma_{0}=A$. Then the normalized material intrinsic length scale is given by

$$
\frac{\bar{l}}{b}\left(\frac{\sigma_{0}}{\mu}\right)^{2}=\left(1+C \ln \dot{\varepsilon}^{*}\right)^{-2}\left(1-T^{* m}\right)^{-2}
$$

This equation gives the material intrinsic length in the presence of both strain rate and temperature 
variation (as shown in Fig. 2 for $C=0.014$ and $m=1$.). By setting $\dot{\varepsilon}^{*}=1.0$ or $T^{*}=0$ in eqn. (7), we can obtain the modification of the temperature variation or strain-rate effect on $\bar{l}$, respectively. As predicted by the Cowper-Symonds constitutive equation, the characteristic material length scale $\bar{l}$ decreases with increasing strain rate; on the other hand, this length scale rises with increasing temperature.

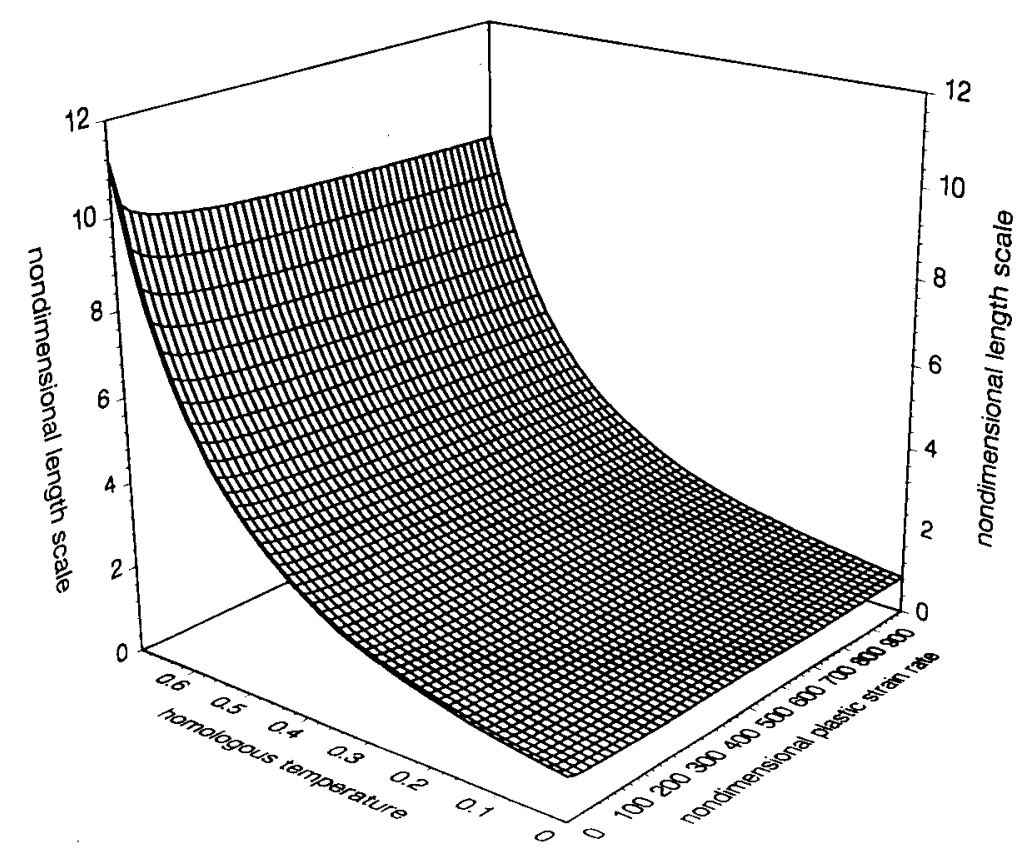

Fig. 2 Variation of nondimensional length scale with both strain rate and temperature changes

\section{CONCLUSION}

For MEMS, there are several kinds of important intrinsic length scales [12-14]: intermolecular spacing of the material, Debye length, thermal mean free path, mean free path of gases, material intrinsic length scales, etc. This paper concerns the dynamic properties of the material intrinsic length.

Cowper-Symonds constitutive equation is used to study the influence of strain rate effect on the characteristic material length scale in strain gradient plasticity. Johnson-Cook constitutive equation is adopted to study the influence of both strain-rate effect and temperature variation on the aforementioned length scale. The characteristic material length scale decreases with increasing strain rates, but it increases with temperature.

\section{ACKNOWLEDGEMENT}

This work is jointly sponsored by the National Natural Science Foundation of China and a key 
project from Chinese Academy of Sciences.

\section{REFERENCES}

1. W. D. Nix and H. Gao, J. Mech. Phys. Solids, 46 (1998) 411-425.

2. Y. P. Zhao. J. Shijiazhuang Railway Institute, 12 (1999) 13-18.

3. K. C. Hwang and H. Q. Jiang, in: Plasticity and its Applications, Z. P. Huang, ed., Beijing Institute of Technology Press, Beijing (1999) pp.31-36.

4. G. R. Cowper and P. S. Symonds, Brown University Division of Applied Mathematics Report, No. 28 (1957) September.

5. S. R. Bodner and P. S. Symonds, J. Appl. Mech., 29 (1962) 719-728.

6. P. S. Symonds and C. T. Chon, in: Mechanical Properties of Materials at High Strain Rates, Institute of Physics Conference Series No.21 (1974) pp. 299-316.

7. M. J. Forrestal and M. J. Sagartz, J. Appl. Mech., 45 (1978) 685-687.

8. G. R. Johnson W. H. Cook, Eng. Fract. Mech., 21 (1985) 31-48.

9. G. R. Johnson and W. H. Cook, in: Proc. $7^{\text {th }}$ Int. Symp. Ballistics (1983) pp.541-547.

10. M. A. Meyers, Dynamic Behavior of Materials. John Wiley \& Sons, Inc. (1994).

11. P. Gardiner, et al, J. Phys. IV France, C3 (1997) 593-597.

12. L. Chang, in: Proc. IMEMS/IMOEMS Res., March 6-7, Shanghai (1999) pp. 3-5.

13. Y. P. Zhao, in: Proc. IMEMS/IMOEMS Res., March 6-7, Shanghai (1999) pp. 129-132.

14. Y. P. Zhao, Mesoscopic mechanical engineering, Science Foundation in China (to appear). 
Advances in Engineering Plasticity

10.4028/www.scientific.net/KEM.177-180

Influences of Strain Rate and Temperature Variation on Material Intrinsic Length of Plasticity Strain Gradient Theory

10.4028/www.scientific.net/KEM.177-180.47 